\title{
Comparison of the clinical characteristics of primary thyroid lymphoma and diffuse sclerosing variant of papillary thyroid carcinoma
}

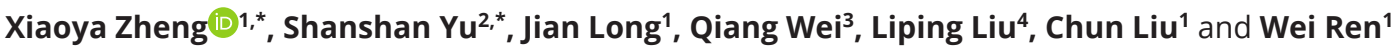 \\ ${ }^{1}$ Department of Endocrinology, The First Affiliated Hospital of Chongqing Medical University, Chongqing, China \\ ${ }^{2}$ Pathology Department, Chongqing Medical University, Chongqing, China \\ ${ }^{3}$ Prevention of Disease Department, Chongqing Jiulongpo District Hospital of Traditional Chinese Medicine, Chongqing, China \\ ${ }^{4}$ Department of Ultrasound, The First Affiliated Hospital of Chongqing Medical University, Chongqing, China
}

Correspondence should be addressed to X Zheng: zxy203405@hospital-cqmu.com

*(X Zheng and S Yu contributed equally to this work)

\begin{abstract}
Objective: Both primary thyroid lymphoma (PTL) and diffuse sclerosing variant of papillary thyroid carcinoma (DSVPTC) are two rare malignant tumours with different therapies and prognoses. This study compared their clinical features.

Methods: From a retrospective review of the pathologic database at our institute between January 2015 and August 2020, 52 PTL patients and 40 DSVPTC patients were included. Demographic, clinical, laboratory and ultrasound data were extracted from electronic medical records. Statistical analyses were performed using GraphPad Prism 5.0. Results: Both PTL and DSVPTC were more likely to occur in women 83.7 and $67.5 \%$, respectively), but DSVPTC patients were younger (median age: 36 vs 64.5), had fewer compressive symptoms, and more frequently had neck lymph node metastasis than PTL patients. The prevalence of Hashimoto's thyroiditis (HT) and hypothyroidism was significantly higher in PTL patients than in DSVPTC patients (31\% vs $17.5 \%$ ). Hyperthyroidism could only be found in DSVPTC patients, which accounted for $7.5 \%$. Heterogeneous echogenicity and irregular edges were frequently observed in both PTL and DSVPTC. However, compared with PTL, DSVPTC exhibited smaller lesion sizes, higher frequencies of diffuse sonographic patterns and calcification and lower frequencies of hypoechoic features and internal blood flow signal. The overall survival rate with PTL was $77.23 \%$, which was lower than that with DSVPTC (90.91\%), but this difference was not significant $(P=0.096)$.

Conclusion: Clinical characteristics such as age, compression symptoms, and sonographic features such as a large mass with heterogeneous echogenicity, hypoechoic, irregular edges, and calcification are helpful for impression diagnosis of PTL and DSVPTC before surgery.
\end{abstract}

\author{
Key Words \\ - primary thyroid lymphoma \\ - diffuse sclerosing variant \\ of papillary thyroid \\ carcinoma \\ - Hashimoto's thyroiditis \\ - ultrasound
}

Endocrine Connections (2022) 11, e210364

\section{Introduction}

Primary thyroid lymphoma (PTL) is a rare malignant tumour that accounts for less than $5.0 \%$ of all malignant thyroid tumours and approximately $2.5-7.0 \%$ of all extranodal lymphomas $(1,2)$. The underlying pathogenesis of PTL remains unclear, but the major risk factor for PTL is the presence of Hashimoto's thyroiditis (HT), which causes a 40- to 80-fold increase in the risk of PTL $(3,4,5,6)$. Diffuse sclerosing variant of papillary thyroid carcinoma (DSVPTC) is also an uncommon tumour making up https://ec.bioscientifica.com https://doi.org/10.1530/EC-21-0364 (c) 2022 The authors Published by Bioscientifica Ltd

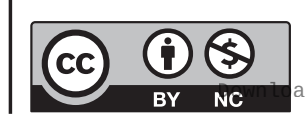

This work is licensed under a Creative Commons Attribution-NonCommercial 4.0 International License. ded from Bioscientifica.com at 04/26/2023 09:10:47AM 
approximately $2 \%$ of all papillary thyroid carcinomas (PTCs) (7). DSVPTC has characteristic nuclear features of PTCs. In addition, carcinoma shows marked squamous metaplasia, numerous psammoma bodies, extensive interstitial fibrosis and heavy lymphocytic infiltration (8). The association of DSVPTC with HT has been reported because both are present with goitre and with heavy infiltration of chronic inflammatory cells (9). The presence of co-existing HT can make the preoperative diagnosis of DSVPTC difficult $(10,11)$.

Both PTL and DSVPTC are lymphocyte-rich thyroid lesions, but the therapeutic methods for these two diseases are different (12). PTL is a potentially curable thyroid malignancy, and the main treatment is multiple periodic chemotherapy (13), while initial radical surgery followed by radioiodine treatment is the common management strategy for DSVPTC (11). Therefore, a correct distinction between these tumours is quite important to avoid unnecessary surgery. To our knowledge, there have been no previous investigations comparing the clinical features between PTL and DSVPTC. Therefore, the aim of the present study was to compare these characteristics.

\section{Research design and methods}

\section{Patients}

From a retrospective review of the pathologic database at our institute between January 2015 and August 2020, 52 patients with PTL and 40 patients with DSVPTC were included in the study. Demographic, clinical and laboratory data were extracted from the electronic medical records. This was a retrospective analysis. The study was approved by the Human Research Ethics Committee of the First Affiliated Hospital of Chongqing Medical University. During the telephone follow-up, consent has been obtained from each patient or relative after full explanation of the purpose and nature of all data used.

\section{Pathologic evaluation}

All the pathologic specimens analysed in this study were reviewed by a pathologist with plentiful experience in thyroid pathology. All diagnoses of thyroid lymphoma are based on the results of pathology and immunohistochemistry, and the diagnosis of some cases also combines genetic testing, such as fluorescence in situ hybridization. The specimens were classified according to the World Health Organization (WHO) classification of lymphomas in 2016 (14). Fine needle aspiration biopsy was defined as a biopsy performed with a needle gauge of $25 \mathrm{G}$, and it was performed under ultrasound guidance. The diagnosis of DSVPTC was made according to the WHO classification, and the disease was characterized by extensive squamous metaplasia, diffuse fibrosis, calcification, abundant lymphocytic infiltration and psammoma bodies (15).

\section{Laboratory test}

The serum levels of triiodothyronine, thyroxine, free triiodothyronine (FT3), free thyroxine (FT4), ultrasensitive thyroid-stimulating hormone (uTSH), thyroid peroxidase antibody (TPOAb), thyroid antithyroglobulin autoantibody (TgAb) and parathyroid hormone were detected by an immunochemical-automated analyser (Beckman Coulter UniCelDxI 800; Beckman Coulter, Inc., Brea, CA, USA). TSH receptor antibody was detected by another immunochemical-automated analyser (Roche Cobas e601; Roche).

\section{Thyroid ultrasonography}

Thyroid sonography was performed on an HD7 ultrasound system (Philips) by experienced technicians who were blind to the design of this study. The ultrasonographic analysis was reviewed by radiologists (with more than 10 years of experience in thyroid imaging diagnosis) without any previous knowledge of the pathologic results. 'Hypoechoic' was defined as having decreased echogenicity relative to adjacent thyroid tissue and 'very hypoechoic' as decreased echogenicity relative to adjacent musculature.

\section{Definition of diseases}

PTL refers to lymphoma with thyroid as the first manifestation, involves the thyroid gland alone, with or without the local lymph node infiltration. Except for cases that have a history of lymphoma or other distant regions, a metastatic spread of lymphoma occurred at the time of the first diagnosis (16). The diagnosis of hyperthyroidism was defined as either having a decreased TSH value $<0.56 \mu \mathrm{IU} / \mathrm{mL}$ or being treated with antithyroid drugs. The presence of hypothyroidism was defined as either having an elevated TSH value $>5.91 \mu \mathrm{IU} / \mathrm{mL}$ or being on levothyroxine replacement therapy at the time of diagnosis. The diagnosis of HT was suggested by a typical ultrasound pattern or by the presence of antithyroid antibodies (17).

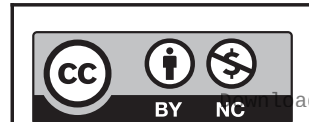




\section{Statistical analysis}

Statistical analyses were performed using GraphPad Prism 5.0. Continuous variables with normal distributions are expressed as mean \pm S.D. and were tested by Student's $t$ test; variables with non-normal distributions are expressed as medians (interquartile range) and were tested by non-parametric testing (Mann-Whitney $U$ test). Categorical variables are described as percentages (\%) and were tested by the chi-square test or Fisher's test. The logrank (Mantel-Cox) test was used to compare the survival curves. All statistical analyses were two-sided, and $P<0.05$ was considered statistically significant.

\section{Results}

Theclinical characteristics of patients with PTLand DSVPTC are summarized in Table 1 . This study retrospectively analysed 52 patients with PTL and 40 patients with DSVPTC were confirmed by surgical pathology. Representative histological figures of PTL and DSVPTC were shown in Fig. 1. The median age at presentation of patients with PTL was 64.5 years, while DSVPTC was more often seen in a younger age group, with a median age at presentation of 36. Both PTL and DSVPTC tended to occur more frequently in women; $83.7 \%$ were females among the 52 patients with PTL, and $67.5 \%$ were females among the 40 patients with DSVPTC. The median duration of PTL was 1 month (interquartile range: $0.42-2.5$ months), while the median duration of DSVPTC was 3.3 months (interquartile range: 1.55-7.25 months). Compression symptoms such as a palpable neck mass, difficulty breathing and dysphagia were more frequent in patients with PTL than in patients with DSVPTC. Symptoms such as neck pain, hoarseness, weight loss and fever were present in two to four patients with PTL, while these symptoms were very rare in patients with DSVPTC. Compared to PTL, DSVPTC had a higher incidence of lymph node metastasis $(82.5 \%$ vs $30.8 \%$, $P<0.001)$. There were no significant differences in radiation exposure history or thyroid cancer family history between the two groups $(P>0.05)$.

In the laboratory findings, there were no significant differences in thyroid hormone levels (including total triiodothyronine (TT3), total thyroxine (TT4), FT3 and FT4) or uTSH levels between the two groups $(P>0.05)$. There were also no significant differences in thyroid autoimmune antibodies (including $\mathrm{Tg}, \mathrm{Tg} \mathrm{Ab}$ and TPOAb) between the two groups $(P>0.05)$; detailed data are shown in Table 2 . When we defined HT as elevated thyroid autoimmune antibody or by the typical ultrasound pattern, we found that the prevalence of HT was much higher in the PTL group than in the DSVPTC group $(65.4 \%$ vs $27.5 \%, P<0.05)$ (Fig. 2A). Three patients (7.5\%) presented with hyperthyroidism combined with DSVPTC, while none were diagnosed with hyperthyroidism and PTL. Hypothyroidism was defined as uTSH- or being on levothyroxine replacement therapy. We found that the prevalence of hypothyroidism in patients with PTL was higher than that in patients with DSVPTC (31\% vs 17.5\%) (Fig. 2B).

The dominant sonographic findings are shown in Table 3. The dominant sonographic findings of PTL were large size (average $5.7 \pm 1.8 \mathrm{~cm}$ ), nodular type $(73.1 \%)$, heterogeneous echogenicity (86.5\%), hypoechoic features $(82.7 \%)$, irregular edges $(90.4 \%)$, increased

Table 1 Clinical characteristics of patients with PTL and DSVPTC. The duration of disease comes from the time between the appearance of the chief complaint in the medical history record and the confirmation of pathological diagnosis.

\begin{tabular}{l}
\hline Characteristics \\
\hline Age (years) \\
Female, $n(\%)$ \\
Duration of disease (months) \\
Radiation exposure history, $n(\%)$ \\
Family history of thyroid cancer, $n(\%)$ \\
Palpable mass, $n(\%)$ \\
Dyspnea, $n(\%)$ \\
Dysphagia, $n(\%)$ \\
Neck pain, $n(\%)$ \\
Hoarseness, $n(\%)$ \\
Weight Loss, $n(\%)$ \\
Fever, $n(\%)$ \\
Metastases, $n(\%)$
\end{tabular}

\begin{tabular}{c} 
PTL $(n=52)$ \\
\hline $64.5(55.25-71.75)$ \\
$43(83.7)$ \\
$1(0.42-2.5)$ \\
$1(1.9)$ \\
$2(3.8)$ \\
$19(36.5)$ \\
$8(15.4)$ \\
$5(9.6)$ \\
$3(5.8)$ \\
$3(5.8)$ \\
$4(7.7)$ \\
$2(3.8)$ \\
$16(30.8)$
\end{tabular}

NC

$\begin{array}{lll}\text { DSV }(n=40) & & \text { P value } \\ 36(31-46.75) & & <0.001^{a} \\ 27(67.5) & & 0.14 \\ 3.3(1.55-7.25) & & 0.23 \\ 2(5) & 0.58 \\ 3(7.5) & & 0.65 \\ 6(15) & 0.03^{a} \\ 2(5) & & 0.18 \\ 1(2.5) & 0.23 \\ 0 & \\ 0 & \\ 0 & \\ 0 & \\ 33(82.5) & \\ \end{array}$

a $P<0.05$, the difference was statistically significant.

PTC, papillary thyroid carcinoma; PTL, primary thyroid lymphoma.

https://ec.bioscientifica.com

https://doi.org/10.1530/EC-21-0364
(C) 2022 The authors Published by Bioscientifica Ltd
This work is licensed under a Creative Commons Attribution-NonCommercial 4.0 International License. ded from Bioscientifica com at 04/26/2023 09:10:47AM 


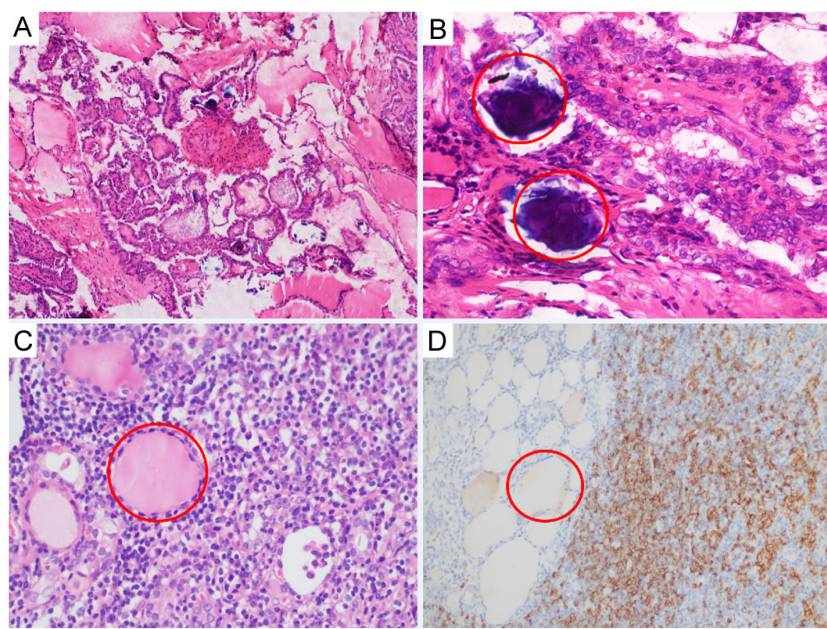

Figure 1

Representative histological figures of diffuse sclerosing variant of papillary thyroid carcinoma (DSVPTC) and thyroid diffuse large B-cell lymphoma (DLBCL). (A) Histopathology of DSVPTC showing the carcinoma had numerous psammoma bodies, papillary structures and fibrous stroma (haematoxylin and eosin, $\times 100$ ). (B) Higher magnification showing that many tumour cells have squamoid differentiation and nuclear characteristic of papillary thyroid carcinoma. Psammoma bodies were in the red circle (haematoxylin and eosin, $\times 400$ ). (C) Representative case with thyroid DLBCL. Thyroid follicles were in the red circle (haematoxylin and eosin, $\times 400$ ). (D) Representative immunostaining for B cell-derived lymphoma with MAB against cell surface marker CD20 $(\times 200)$. Thyroid follicles were in the red circle.

Table 2 Laboratory findings of patients with PTL and DSVPTC.

\begin{tabular}{l} 
Variable \\
\hline TT3 $(0.66-1.61 \mathrm{ng} / \mathrm{mL})$ \\
TT4 $(5.44-11.85 \mu \mathrm{g} / \mathrm{dL})$ \\
FT3 $(2.01-4.82 \mathrm{pg} / \mathrm{mL})$ \\
FT4 $(0.59-1.25 \mathrm{ng} / \mathrm{dL})$ \\
UTSH $(0.56-5.91 \mu \mathrm{lU} / \mathrm{mL})$ \\
$\operatorname{Tg}(0-50.03 \mathrm{ng} / \mathrm{mL})$ \\
$\operatorname{TgAb}(0-4 \mathrm{IU} / \mathrm{mL})$ \\
$\operatorname{TPOAb}(0-9 \mathrm{IU} / \mathrm{mL})$ \\
$\operatorname{TRAb}(0.3-1.8 \mathrm{IU} / \mathrm{L})$ \\
\end{tabular}

\begin{tabular}{c}
\hline PTL $(n=52)$ \\
\hline $0.89 \pm 0.20$ \\
$8.40 \pm 1.51$ \\
$2.93 \pm 0.72$ \\
$0.61 \pm 0.36$ \\
$4.91(2.57-48.8)$ \\
$12.3(4.18-81.12)$ \\
$25(6.55-1322)$ \\
$56.9(25.3-668.1)$ \\
$0.4(0.3-1.4)$ \\
\hline
\end{tabular}

\begin{tabular}{c}
\hline \multicolumn{1}{c}{ DSV $(n=40)$} \\
\hline $0.87 \pm 0.19$ \\
$6.99 \pm 2.21$ \\
$2.89 \pm 0.66$ \\
$0.86 \pm 0.23$ \\
$2.29(1.64-12.48)$ \\
$18.4(3.16-88.56)$ \\
$8.5(2.5-1002)$ \\
$23.2(1.6-316.6)$ \\
$1.5(0.6-2.2)$ \\
\hline
\end{tabular}

\begin{tabular}{c}
\hline P value \\
\hline 0.79 \\
0.28 \\
0.62 \\
0.71 \\
0.54 \\
0.94 \\
0.11 \\
0.13 \\
0.58 \\
\hline
\end{tabular}

FT3, free triiodothyronine; FT4, free thyroxine; PTC, papillary thyroid carcinoma; PTL, primary thyroid lymphoma; TgAb, antithyroglobulin autoantibodies; TPOAb, antithyroperoxidase autoantibodies; TRAb, TSH receptor autoantibodies; TT3, total triiodothyronine; TT4, total thyroxine; uTSH, ultrasensitive thyroid-stimulating hormone.

internal vascularity (84.6\%) and calcifications (1.9\%). DSVPTC cases mainly presented with diffuse type (85\%), heterogeneous echogenicity (97.5\%), irregular edges (87.5\%) and calcifications (100\%). Hypoechoic vascularity (22.5\%) and increased internal vascularity (20\%) were not commonly seen in patients with DSVPTC.

For analysis of the overall survival rate, data on patients who were alive at the last follow-up contact were censored.

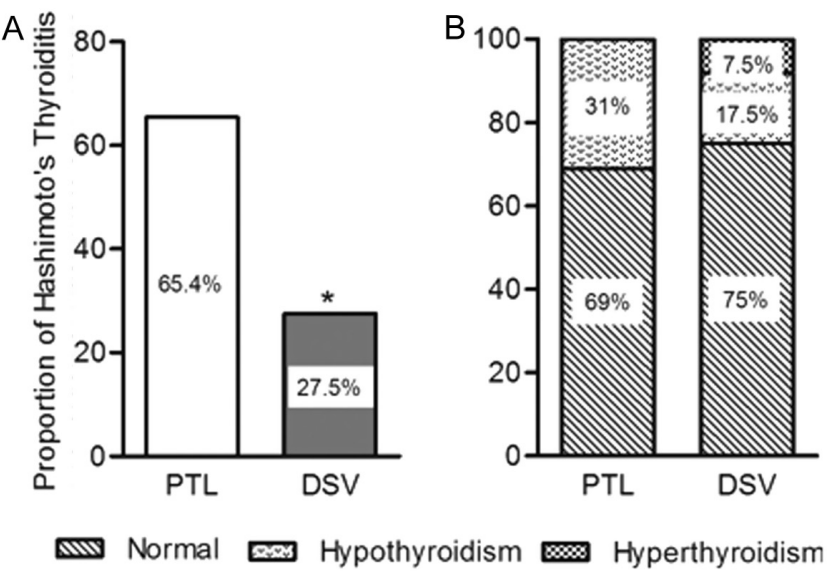

\section{Figure 2}

Comparison of the percentage of Hashimoto's thyroiditis (HT) cases and the proportion of different thyroid functions in patients with primary thyroid lymphoma (PTL) and diffuse sclerosing variant of papillary thyroid carcinoma. (A) The diagnosis of HT is suggested by a typical ultrasound pattern or by the presence of antithyroid antibodies. (B) Hyperthyroidism was defined as either an ultrasensitive thyroid-stimulating hormone (UTSH) value $<0.56 \mu \mathrm{lU} / \mathrm{mL}$ or treatment with antithyroid drugs. Hypothyroidism was defined as UTSH $>5.91 \mu \mathrm{IU} / \mathrm{mL}$ or being on levothyroxine replacement therapy.

The overall survival rate of patients with PTL was $77.23 \%$ (95\% CI, 53.15-89.98\%), and that of patients with DSVPTC was 90.91\% (95\% CI, 50.79-98.77\%) (Fig. 3). There were no significant differences between survival rates in the two diseases $(P=0.096)$. Six patients with PTL died during the follow-up. Lymphoma progression was observed in 3 of the 52 patients due to abandonment therapy; one patient died of pneumonia, and one died of acute heart failure. Information regarding the cause of death was not available for the remaining patient. The only patient with DSVPTC died of thyroid cancer lung metastasis with infection and respiratory failure.

\section{Discussion}

PTL occurs preferentially in females, and the female-tomale ratio is approximately 3:1. The most common clinical presentation of PTL is a rapidly enlarging, painless goitre, which may cause dysphagia, dyspnoea and hoarseness. In addition, B symptoms, including fever, night sweats and weight loss, can be presented $(18,19,20)$. The most frequent cases include non-Hodgkin lymphoma derived from B cells, mainly diffuse large B-cell lymphoma followed by mucosaassociated lymphoid tissue (MALT) lymphoma (21).

DSVPTC is characterized by diffuse enlargement of the thyroid gland and often lacks a specific mass (13). DSVPTC tends to occur more frequently in women, and the male-

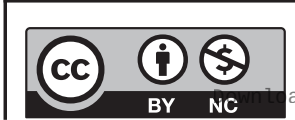

This work is licensed under a Creative Commons Attribution-NonCommercial 4.0 International License. ded from Bioscientifica.com at 04/26/2023 09:10:47AM 
Table 3 Ultrasound features of PTL and DSVPTC.

\begin{tabular}{l}
\hline Variable \\
\hline Size (maximum diameter of lesion, $\mathrm{cm})$ \\
Sonographic pattern, $n(\%)$ \\
$\quad$ Diffuse, $n(\%)$ \\
$\quad$ Nodular, $n(\%)$ \\
Heterogeneous echogenicity, $n(\%)$ \\
Hypoechoic, $n(\%)$ \\
Irregular edge, $n(\%)$ \\
Increased internal vascularity, $n(\%)$ \\
Calcifications, $n(\%)$
\end{tabular}

\begin{tabular}{c}
\hline PTL $(n=52)$ \\
\hline $5.7 \pm 1.8$ \\
$14(26.9)$ \\
$38(73.1)$ \\
$45(86.5)$ \\
$43(82.7)$ \\
$47(90.4)$ \\
$44(84.6)$ \\
$1(1.9)$
\end{tabular}

\begin{tabular}{c}
\hline $\mathbf{D S V}(n=40)$ \\
\hline $4.2 \pm 0.6$ \\
$34(85)$ \\
$6(15)$ \\
$39(97.5)$ \\
$9(22.5)$ \\
$35(87.5)$ \\
$8(20)$ \\
$40(100)$ \\
\hline
\end{tabular}

\begin{tabular}{c}
\hline P value \\
\hline $0.007^{a}$ \\
$<0.001^{a}$ \\
0.06 \\
$<0.001^{a}$ \\
0.19 \\
$<0.001^{a}$ \\
$<0.001^{a}$
\end{tabular}

a $P<0.05$, the difference was statistically significant.

PTC, papillary thyroid carcinoma; PTL, primary thyroid lymphoma.

to-female ratio is approximately 1:5. Hyper-echogenicity, diffuse-scattered microcalcification and cervical lymph node metastasis are the characteristic ultrasonographic features of DSVPTC (9). On microscopic examination, DSVPTC has characteristic nuclear features of PTC. In addition, the carcinoma shows marked squamous metaplasia, numerous psammoma bodies, extensive interstitial fibrosis and heavy lymphocytic infiltration. Additionally, DSVPTC shows a higher prevalence of extra-thyroidal extension and cervical lymph node metastasis $(10,12)$.

Typical DSVPTC shows different histological characteristics from PTL. For this reason, it is not difficult to diagnose these pathologies on microscopic examination. However, PTL and DSVPTC are both lymphocyte-rich thyroid lesions, and diagnostic challenges occur when these two types of malignancies are associated in the

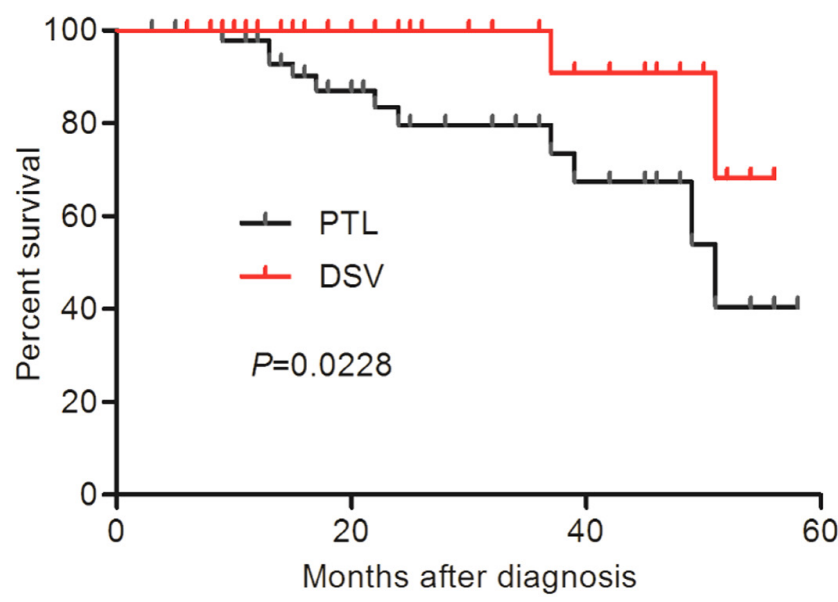

Figure 3

Comparison of the survival rate of patients with primary thyroid lymphoma (PTL) (black line) and diffuse sclerosing variant of papillary thyroid carcinoma (DSVPTC) (red line). The overall survival rate of PTL patients was $77.23 \%(95 \% \mathrm{Cl}, 53.15-89.98 \%)$ and that of DSVPTC patients was $90.91 \%$ (95\% Cl, 50.79-98.77\%). context of HT (22). Sometimes, PTL or DSVPTC can be mistaken clinically for thyroiditis because they share the same appearance of inflammatory background (9). The development of thyroidal MALT lymphoma has been described as an adverse event in patients suffering from long-standing HT (23). Differently, autoimmune thyroiditis is included among independent risk factors for papillary thyroid cancer development (24). It is important to differentiate PTL from DSVPTC, as these diseases carry different prognoses and different treatment strategies. Both PTL and DSVPTC have generally good prognoses if diagnosed early and accurately and treated appropriately. Therefore, early preoperative diagnosis is helpful to develop a treatment strategy and improve the prognosis. The preoperative diagnosis of thyroid tumours depends on clinical symptoms and signs, laboratory examinations and ultrasound imaging examinations. When comparing the clinical features, we found that both PTL and DSVPTC were more likely to occur in women, but patients with DSVPTC were younger, had fewer compressive symptoms and more frequently had neck lymph node metastasis than patients with PTL. This may be due to the fact that PTL is characterized by a rapidly growing mass in the neck, which may cause compression symptoms (18), while most DSVPTCs usually have indolent characteristics (25).

The laboratory findings did not show significant differences in thyroid hormone and autoimmune antibody levels between patients with PTL or DSVPTC. However, the prevalence of HT and hypothyroidism was significantly higher in patients with PTL than in those with DSVPTC (31\% vs $17.5 \%$ ). Hyperthyroidism could only be found in patients with DSVPTC, which accounted for $7.5 \%$. HT is the most common form of autoimmune thyroiditis with heterogeneous clinical and pathological characteristics. In recent years, there have been case reports in the literature of PTL and DSVPTC occurring concurrently with HT. Some studies have indicated that both PTL and DSVPTC are

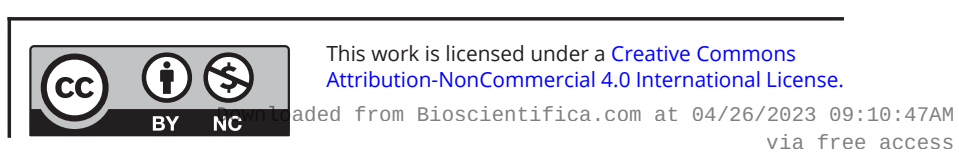


complications of HT $(3,26)$. The potential mechanism has generally been explained by aberrant follicular epithelial regeneration following chronic inflammatory damage or by the possibility that HT harbours potential precursor lesions of thyroid cancer (27). This evidence strongly supports a causal relationship between thyroid cancer and pre-existing HT, and HT is considered a preneoplastic condition promoting thyroid carcinogenesis (28). In any case, in the context of HT, the risks of PTL and DSVPTC are significantly increased, and the difficulty of diagnosis is also significantly increased. Therefore, we should pay more attention to the differential diagnosis of PTL and DSVPTC when Hashimoto coexists.

Ultrasonography has been proven to be the most efficient method for the diagnosis of thyroid diseases and has the advantages of being non-invasive, involving realtime scanning, being easy to perform and being widely available (13). When comparing the ultrasound features of PTL and DSVPTC, we found that both PTL and DSVPTC had heterogeneous echogenicity and irregular edges, but the lesion sizes of DSVPTC were smaller, diffuse sonographic patterns and calcifications were more frequently seen, and hypoechoic appearance and increased internal vascularity were rarer than those of PTL. The ultrasound features of PTL and DSVPTC were consistent with the findings of previous studies $(13,29)$.

The overall survival rate of PTL patients was $77.23 \%$, which was lower than that of patients with DSVPTC (90.91\%). Although the survival rate between the two groups was not significantly different due to the small sample size, the mortality rate of PTL was still slightly higher. The high mortality of patients with PTL might be related to their malignant histological types, advanced age, many complications and failure to receive active treatments (20). In contrast, although most patients with DSVPTC have cervical lymph node metastasis at the beginning of diagnosis, surgery and radiation therapy are very effective, and the patients are relatively young and generally have a better prognosis (13).

There are some limitations to this study. First, we had a small population size for PTL and DSVPTC because both were rare diseases, and it was difficult to obtain many cases for the analysis. Secondly, we only retrospectively analysed patients with medical records in our hospital for the past 5 years, and the data are not broadly representative. Thirdly, this is a retrospective study. Although both PTL and DSVPTC are related to HT, they cannot explain the causal relationship between HT and these two diseases. Further multi-centre, large-sample, prospective research studies are needed to explore the relationship between HT and these thyroid tumours.
Both PTL and DSVPTC are lymphocyte-rich thyroid lesions, and sometimes it is difficult to differentially diagnose such patients with HT before surgery. In this retrospective study, we found certain clinical features, such as age, duration, compression symptoms; sonographic features such as size of thyroid or mass, echogenicity, irregular edges and calcifications were helpful for initial impression diagnosis of PTL and DSVPTC before surgery. Further differential diagnosis and confirmation will depend on the combination of cytology, histology and even genetics.

\section{Declaration of interest}

The authors declare that there is no conflict of interest that could be perceived as prejudicing the impartiality of the research reported.

\section{Funding}

This project was supported by the National Natural Science Foundation of China Youth Program (Grant No. 81900733), the Chongqing Municipal Health and Family Planning Commission Fund (Number: 2021MSXM013), Bethune Charity Foundation (G-X-2020-1107-14) and the National Key Clinical Specialties Construction Program of China in 2011.

\section{Ethics approval}

This was a retrospective analysis study. The study was approved by the Human Research Ethics Committee of the First Affiliated Hospital of Chongqing Medical University.

\section{References}

1 Walsh S, Lowery AJ, Evoy D, McDermott EW \& Prichard RS. Thyroid lymphoma: recent advances in diagnosis and optimal management strategies. Oncologist 201318 994-1003. (https://doi.org/10.1634/ theoncologist.2013-0036)

2 Stein SA \& Wartofsky L. Primary thyroid lymphoma: a clinical review. Journal of Clinical Endocrinology and Metabolism 201398 3131-3138. (https://doi.org/10.1210/jc.2013-1428)

3 Ahmed T, Kayani N, Ahmad Z \& Haque MN. Non-Hodgkin's thyroid lymphoma associated with Hashimoto's thyroiditis. Journal of the Pakistan Medical Association 201464 342-344.

4 Kim NR, Ko YH \& Lee YD. Primary T-cell lymphoma of the thyroid associated with Hashimoto's thyroiditis, histologically mimicking MALT-lymphoma. Journal of Korean Medical Science 201025 481-484. (https://doi.org/10.3346/jkms.2010.25.3.481)

5 Levy-Blitchtein S, Plasencia-Rebata S, Morales Luna D \& Del Valle Mendoza J. Coexistence of papillary thyroid microcarcinoma and mucosa-associated lymphoid tissue lymphoma in a context of Hashimoto's thyroiditis. Asian Pacific Journal of Tropical Medicine 2016 9 812-814. (https://doi.org/10.1016/j.apjtm.2016.06.017)

6 Roldan-Valadez E, Ortega-Lopez N, Cervera-Ceballos E, ValdiviesoCardenas G, Vega-Gonzalez I \& Granados-Garcia M. Whole-body (18)F-FDG PET/CT in primary non-Hodgkin's lymphoma of the thyroid associated with Hashimoto's thyroiditis and bilateral kidney infiltration. Revista Española de Medicina Nuclear 200827 34-39. (https://doi.org/10.1157/13114368)

7 Thompson LD, Wieneke JA \& Heffess CS. Diffuse sclerosing variant of papillary thyroid carcinoma: a clinicopathologic and

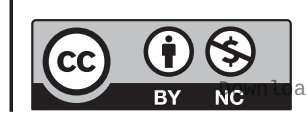

This work is licensed under a Creative Commons Attribution-NonCommercial 4.0 International License. Atrom Bioscientifica com at 04/26/2023 09:10:47AM 
immunophenotypic analysis of 22 cases. Endocrine Pathology 200516 331-348. (https://doi.org/10.1385/ep:16:4:331)

8 Lee JY, Shin JH, Han BK, Ko EY, Kang SS, Kim JY, Oh YL \& Chung JH. Diffuse sclerosing variant of papillary carcinoma of the thyroid: imaging and cytologic findings. Thyroid 200717 567-573. (https://doi. org/10.1089/thy.2006.0321)

9 Chen CC, Chen WC, Peng SL \& Huang SM. Diffuse sclerosing variant of thyroid papillary carcinoma: diagnostic challenges occur with Hashimoto's thyroiditis. Journal of the Formosan Medical Association 2013112 358-362. (https://doi.org/10.1016/j.jfma.2012.07.020)

10 Takagi N, Hirokawa M, Nobuoka Y, Higuchi M, Kuma S \& Miyauchi A. Diffuse sclerosing variant of papillary thyroid carcinoma: a study of fine needle aspiration cytology in 20 patients. Cytopathology 201425 199-204. (https://doi.org/10.1111/cyt.12076)

11 Pillai S, Gopalan V, Smith RA \& Lam AK. Diffuse sclerosing variant of papillary thyroid carcinoma - an update of its clinicopathological features and molecular biology. Critical Reviews in Oncology/Hematology 201594 64-73. (https://doi.org/10.1016/j.critrevonc.2014.12.001)

12 Baloch ZW \& LiVolsi VA. Fine-needle aspiration cytology of papillary Hurthle cell carcinoma with lymphocytic stroma 'Warthin-like tumor' of the thyroid. Endocrine Pathology 19989 317-323. (https://doi. org/10.1007/BF02739691)

13 Gu LS, Cui NY, Wang Y, Che SN, Zou SM, He W, Liu JY \& Gong XT. Comparison of sonographic characteristics of primary thyroid lymphoma and anaplastic thyroid carcinoma. Journal of Thoracic Disease 20179 4774-4784. (https://doi.org/10.21037/jtd.2017.09.48)

14 Cazzola M. Introduction to a review series: the 2016 revision of the WHO classification of tumors of hematopoietic and lymphoid tissues. Blood 2016127 2361-2364. (https://doi.org/10.1182/blood-2016-03657379)

15 Vuong HG, Kondo T, Pham TQ, Oishi N, Mochizuki K, Nakazawa T, Hassell L \& Katoh R. Prognostic significance of diffuse sclerosing variant papillary thyroid carcinoma: a systematic review and metaanalysis. European Journal of Endocrinology 2017 176 433-441. (https:// doi.org/10.1530/EJE-16-0863)

16 Pavlidis ET \& Pavlidis TE. A review of primary thyroid lymphoma: molecular factors, diagnosis and management. Journal of Investigative Surgery 201932 137-142. (https://doi.org/10.1080/08941939.2017.1383 536)

17 Radetti G. Clinical aspects of Hashimoto's thyroiditis. Endocrine Development 201426 158-170. (https://doi.org/10.1159/000363162)

18 Graff-Baker A, Roman SA, Thomas DC, Udelsman R \& Sosa JA. Prognosis of primary thyroid lymphoma: demographic, clinical, and pathologic predictors of survival in 1,408 cases. Surgery 2009146 1105-1115. (https://doi.org/10.1016/j.surg.2009.09.020)

19 Sharma A, Jasim S, Reading CC, Ristow KM, Villasboas Bisneto JC, Habermann TM, Fatourechi V \& Stan M. Clinical presentation and diagnostic challenges of thyroid lymphoma: a cohort study. Thyroid 201626 1061-1067. (https://doi.org/10.1089/thy.2016.0095)

20 Watanabe N, Narimatsu H, Noh JY, Iwaku K, Kunii Y, Suzuki N, Ohye H, Suzuki M, Matsumoto M, Yoshihara A, et al. Long-term outcomes of 107 cases of primary thyroid mucosa-associated lymphoid tissue lymphoma at a single medical institution in Japan. Journal of Clinical Endocrinology and Metabolism 2018103 732-739. (https://doi. org/10.1210/jc.2017-01478)

21 Derringer GA, Thompson LD, Frommelt RA, Bijwaard KE, Heffess CS \& Abbondanzo SL. Malignant lymphoma of the thyroid gland: a clinicopathologic study of 108 cases. American Journal of Surgical Pathology 200024 623-639. (https://doi.org/10.1097/00000478-200005000-00001)

22 Trovato M, Giuffrida G, Seminara A, Fogliani S, Cavallari V, Ruggeri RM \& Campennì A. Coexistence of diffuse large B-cell lymphoma and papillary thyroid carcinoma in a patient affected by Hashimoto's thyroiditis. Archives of Endocrinology and Metabolism 2017 61 643-646. (https://doi.org/10.1590/2359-3997000000313)

23 Troch M, Woehrer S, Streubel B, Weissel M, Hoffmann M, Müllauer L, Chott A \& Raderer M. Chronic autoimmune thyroiditis (Hashimoto's thyroiditis) in patients with MALT lymphoma. Annals of Oncology 200819 1336-1339. (https://doi.org/10.1093/annonc/mdn049)

24 Ferrari SM, Fallahi P, Elia G, Ragusa F, Ruffilli I, Paparo SR \& Antonelli A. Thyroid autoimmune disorders and cancer. Seminars in Cancer Biology 202064 135-146. (https://doi.org/10.1016/j.semcancer.2019.05.019)

25 Ito Y, Kudo T, Kihara M, Takamura Y, Kobayashi K, Miya A \& Miyauchi A. Prognosis of low-risk papillary thyroid carcinoma patients: its relationship with the size of primary tumors. Endocrine Journal 201259 119-125. (https://doi.org/10.1507/endocrj.ej11-0288)

26 Cheng V, Brainard J \& Nasr C. Co-occurrence of papillary thyroid carcinoma and primary lymphoma of the thyroid in a patient with long-standing Hashimoto's thyroiditis. Thyroid 201222 647-650. (https://doi.org/10.1089/thy.2011.0228)

27 Molnar C, Molnar S, Bedekovics J, Mokanszki A, Gyory F, Nagy E \& Méhes G. Thyroid carcinoma coexisting with Hashimoto's Thyreoiditis: clinicopathological and molecular characteristics clue up pathogenesis. Pathology Oncology Research 201925 1191-1197. (https:// doi.org/10.1007/s12253-019-00580-w)

28 Gasbarri A, Sciacchitano S, Marasco A, Papotti M, Di Napoli A, Marzullo A, Yushkov P, Ruco L \& Bartolazzi A. Detection and molecular characterisation of thyroid cancer precursor lesions in a specific subset of Hashimoto's thyroiditis. British Journal of Cancer 200491 1096-1104. (https://doi.org/10.1038/sj.bjc.6602097)

29 Thieblemont C, Mayer A, Dumontet C, Barbier Y, Callet-Bauchu E, Felman P, Berger F, Ducottet X, Martin C, Salles G, et al. Primary thyroid lymphoma is a heterogeneous disease. Journal of Clinical Endocrinology and Metabolism 200287 105-111. (https://doi. org/10.1210/jcem.87.1.8156)

Received in final form 11 December 2021

Accepted 22 December 2021

Accepted Manuscript published online 23 December 2021 https://ec.bioscientifica.com https://doi.org/10.1530/EC-21-0364 (c) 2022 The authors Published by Bioscientifica Ltd

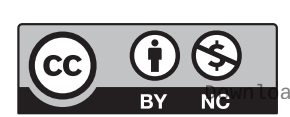

This work is licensed under a Creative Commons Attribution-NonCommercial 4.0 International License. ded from Bioscientifica.com at 04/26/2023 09:10:47AM 